\title{
ITALIANISMI NELLA PROSA NON NARRATIVA CROATA CONTEMPORANEA (sulle opere di Željka Čorak)
}

1. L'autrice di cui qui ci occupiamo è una delle personalità più illustri della cultura croata attuale: storica dell'arte, specialista dell'architettura e dell'urbanesimo della Zagabria otto- e novecentesca, Željka Čorak è anche letterata, saggista, traduttrice, critica e vicepresidente del P.E.N. croato. Tra le sue opere spicca un libro di ricordi intitolato Krhotine ('Frantumi'), ${ }^{1}$ vero capolavoro del genere, tanto artisticamente raffinato quanto profondamente vissuto e umano, dedicato dalla prima all'ultima pagina all'evocazione delle case distrutte nella II guerra mondiale (1942), alle tristi vicende successive e alla descrizione dei mille cimeli ricuperati. ${ }^{2}$ Il volume, uscito nel 1991, è il tentativo dell'autrice di ricomporre i «frantumi», quasi i tasselli di un mosaico vivo nella memoria; una specie della Recherche du temps perdu dei nostri giorni, in sostanza Le temps retrouvé. Vi troviamo tutte le componenti dello stile di Željka Čorak presenti anche negli altri suoi scritti: intellettualismo meditativo e raffinato, vasta erudizione, abbondanza di reminiscenze (non soltanto letterarie), espressione deliberatamente indiretta, mai piatta né triviale, assenza totale di qualsiasi «marcia a vuoto», pregnanza di idee, ricchezza di associazioni. Nelle sue Krhotine vi si aggiunge una commovente affettività e una nobiltà d'animo, priva di qualunque rancore malgrado le tragedie sofferte. Con questi caratteri dello stile concorda la lingua, ricca, moderna e impressiva, lingua che non rifugge da tecnicismi, regionalismi, dialettismi e voci straniere.

La cultura dell'autrice e i principali dominî della sua attività (storia dell'arte, letteratura) permettono di prevedere la presenza di italianismi: infatti, dopo il francese, l'italiano è la lingua neolatina meglio rappresentata nei suoi scritti. ${ }^{3}$ Le pagine che

1 Il sottotitolo Prilog poznavanju hrvatske provincije $u$ devetnaestom stoljeću ('Contributo alla conoscenza della provincia croata nell' Ottocento') allude al principale scopo dell'opera: dimostrare, cioè, che la provincia croata nel secolo scorso non era, come generalmente si crede, esclusivamente rurale, bensì che partecipava in varia misura anche alle correnti culturali dell'epoca (biblioteche private, arti figurative, innovazioni tecniche ecc.).

2 La sezione centrale del libro (pp. 71-202) reca il titolo Među stvarima i snom ('Tra cose e sogno') e consiste nella presentazione dell'inventario ricuperato dalle due case distrutte: oggetti d'uso quotidiano, soprammobili, fotografie. Quasi tutti i capitoli di questa sezione hanno la stessa struttura: una breve descrizione in termini tecnico-artistici (corredata di meravigliose fotografie color seppia) serve da base per evocazioni, reminiscenze piene di affetto e di nostalgia, citazioni, allusioni e via dicendo. 
seguono si dedicano alle voci e frasi italiane in cinque opere di Željka Čorak, cioè nel citato volume Krhotine e in altri quattro libri, che presentiamo nel paragrafo seguente. Va precisato che gli italianismi non appartengono soltanto all'italiano standard ma anche al livello dialettale: la regione dalla quale trae origine la famiglia dell'autrice, il suo Piccolo mondo antico, è la parte più settentrionale del Gorski Kotar, per la precisione i due piccoli centri di Prezid e Čabar, al confine con la Slovenia e non lontano da Rijeka (Fiume). Vi si avvertono da sempre influssi italiani irradiati dall'Istria e dal Veneto, e a queste correnti si deve il manipolo di parole venete nel corpus.

2. Oltre al citato capolavoro, abbiamo spogliato i seguenti libri di Željka Čorak: $\left.{ }^{4} 1\right)$ U funkciji znaka. Drago Ibler i hrvatska arhitektura izmedu dva rata ('In funzione del segno. Drago Ibler e l'architettura croata fra le due guerre'): tesi di laurea (dottorato) dell'autrice, interpretazione in chiave semiotica della personalità e dell'operato dell' architetto croato citato, con ampi sguardi sull' architettura e sulla cultura in genere del detto periodo; 2) Zagrebačka katedrala ('La cattedrale di Zagabria'), in collaborazione con la storica dell'arte Ana Deanović; Željka Čorak vi firma il capitolo sull'Ottocento e sulla ricostruzione della cattedrale dopo il terremoto del $1880 ; 3$ ) Oproštajno pismo gospodinu Mitterrandu ('Lettera d'addio al signor Mitterrand'): otto saggi politici (il quarto è l'eponimo del libro) di scottante attualità, pieni di amarezza e di sarcasmo, a proposito della recente guerra in Croazia e in Bosnia; 4) Zagreb, pisani prostor ('Zagabria, lo spazio scritto'): poco meno di una ventina di testi di argomento architettonico e urbanistico scritti in precedenza (1976-1989), sulla pianificazione urbanistica della città e su certi suoi monumenti (tra i quali la cattedrale ed il cimitero di Mirogoj, entrambi opera dell'architetto Hermann Bollé). Il nostro corpus supera di poco 700 pagine di testo e può dunque essere rappresentativo della lingua e dello stile di Željka Čorak. Data l'importanza dell'Italia nelle arti figurative, è scontato che una notevole parte degli italianismi appartiene alla terminologia artistica, ma elementi linguistici italiani ricorrono anche in ben altri settori lessicali. Tutti gli italianismi,

3 Gli altri idiomi romanzi sono una quantité négligeable: ad. es. rekonkvista (adattamento dello spagnolo reconquista), nel sintagma srpska rekonkvista ('riconquista serba') (OPGM 34). Per completare il quadro aggiungiamo un elemento lessicale che non è beninteso usato come romanismo cosciente bensì come dialettismo, ma che tuttavia è un relitto dell'antico strato neolatino autoctono. Nelle reminiscenze delle scampagnate di una volta si menziona come meta delle gite takozvani »Mašun « ('il cosiddetto «Mašun»') ( $\mathrm{K}$ 284). La qualifica di 'cosiddetto' sembra indicare un appellativo, mentre la maiuscola suggerisce che abbiamo da fare ad un toponimo. Poiché non ci sono altre indicazioni, la localizzazione precisa appare difficile: comunque, è probabile che si tratti del villaggio Mašun, a nord est di Ilirska Bistrica e ad ovest di Prezid. Se è così, e dato che pure in certi altri dialettismi slavi citati nelle Krhotine appare la /a/ per la /o/ protonica, il top. Mašun è probabilmente identico a Mošun, addotto da $\mathrm{P}$. Skok come nome di un villaggio su per giù nella stessa regione (ERHSJ, s.v. mošun). Entrambe le forme sono riflessi del tardolatino MANSIONE (cfr. REW 5311).

4 Per i dati bibliografici completi e per le sigle usate nelle citazioni si veda la bibliografia, sezione A. Le traduzioni in italiano, tra parentesi tonde, sono nostre; le parti del testo omesse sono indicate con tre punti fra parentesi quadre. 
anche quelli puramente tecnici, hanno oltre alla denotativa anche altre funzioni: affettive, pragmatiche, testuali. Si tratta in prevalenza di vocaboli e frasi dell'italiano standard, mentre i dialettismi sono in numero nettamente inferiore.

3. Iniziamo la nostra rassegna con i tecnicismi delle arti figurative, che sono la componente più frequente.

3.1. inkarnat ('incarnato'): il nome viene usato a due riprese. La prima volta (K 144) ha funzione puramente descrittiva, nella presentazione di una statuetta i cui «toni vanno dall'incarnato al grigiastro e al brunastro». La seconda occorrenza, invece ( $\mathrm{K}$ 198), è piena di affettività e di ricordi commoventi. Dopo il II conflitto mondiale la famiglia dell' autrice fu costretta a vendere la sola casa (delle tre) rimasta, e i nuovi proprietari fecero ridipingere le stanze sicché scomparvero per sempre gli ornamenti sulle pareti e sui soffitti, cari ricordi dell'infanzia:

1) za mene je nestao prostor djetinjstva, moja crtana uspavanka: kite cvijeća na zidu, slikani vijenci na stropu, »inkarnat« kuće koji je bio tako neponovljivo bijel $i$ krhak kao neko plemenito staro lice. ('per me sparì lo spazio dell'infanzia, la mia ninnananna disegnata: mazzi di fiori sulle pareti, corone dipinte sul soffitto, l' «incarnato» della casa che era così irripetibilmente bianco e fragile come un vecchio volto nobile.')

La calda affettività risulta dall'accezione figurata del nome incarnato (cfr. le virgolette), dalla personificazione della casa e dal contrasto fra il significato comune di incarnato 'colore rosa carne' (Zingarelli 1992, s. v.) ed il biancore delle camere di una volta.

3.2. palmete ('palmette') (K 148): è la parte degli omamenti di un antico divano coperto di velluto con palmette. La voce fa parte della descrizione tecnica, ma segue una pagina piena di ricordi perché anche questo divano appartiene all'inventario salvato dall'incendio del 1942.

3.3. sagoma ("sagoma') (K 152): nel capitolo su un tavolo ricuperato dal fuoco e poi restaurato si legge:

2) Iz sagome njegove ploče, iz krivulje njegovih nogu još se dadu protumačiti zasade baroknog doba. ('Dalla sagoma della sua tavola, dalla curva delle sue gambe si possono dedurre ancora i princìpi dell'età barocca.').

3.4. stucco-dekoracija ('decorazione a stucco') (K 160): il termine ricorre nella descrizione di una scultura di angeli.

3.5. volute ('volute') (K 188): anche qui si tratta della descrizione tecnica di un bicchiere ornato. La voce compare anche nella descrizione del portale dell'antica cattedrale di Zagabria, distrutta nel 1880, con la qualifica di 'barocca' (barokne volute, ZK 296).

3.6. loggie ('loggie') (ZPP 56): il nome, unito a prozori 'finestre', figura nell'analisi architettonica di uno stabile caratterizzato da linee armoniose e discrete. 
3.7. «pittura metafisica» (ZPP 78; virgolette di Ž. Č.): nel suggestivo capitolo sul crematorio di Zagabria si descrive la sua piramide di vetro e le nuvole che «attraversandola» diventano una 'esemplare «pittura metafisica»'. Secondo Devoto-Oli 1971 questo sintagma denota una tendenza artistica «fondata sull' accostamento inconsueto di oggetti e personaggi e sulla ricerca di una suggestione magica come risultante della loro coesistenza nello spazio», significato che nel testo calza a perfezione.

4. Parole italiane di significato generale si trovano in tutto il nostro corpus. Così, ad esempio, la vigilia di Natale si mettevano nelle finestre i lumini (K 288; nei ricordi della madre di Željka Čorak, che servono da fonte all' autrice e sono incorporati nel libro alle pp. 283-297); i sintagmi anni venti, anni trenta (UFZ 12) si citano come esempio della tendenza attuale a designare i fenomeni artistici riunendoli sotto i relativi decenni; lo spiritosissimo sintagma »vero skai« (ZPP 98; virgolette dell' autrice) è una citazione ironica, paragone di certe costruzioni moderne che deturpano e distruggono gli ultimi spazi liberi della zona residenziale di Zagabria diventando così un surrogato dell' ex-elitismo, proprio dell' architettura del periodo precedente.

5. Meritano una menzione a parte due citazioni letterarie, ambedue con chiara connotazione personale ed affettiva.

5.1. Il primo esempio si legge nel libro sulla cattedrale (ZK 269), e precisamente nelle pagine che illustrano l'attività del noto architetto zagabrese Hermann Bollé, di origine tedesca, benemerito di una serie di edifici e monumenti zagabresi, tra cui spicca la nuova cattedrale (gotica). Criticato per decenni, H. Bollé viene oggi riabilitato e rivalutato, non all'ultimo posto grazie proprio alla nostra autrice. In un passo (a proposito di un ballo solenne) si legge:

3) Umjesto u ulozi gladnog stvaraoca ovisnog o arogantnoj narudžbi, kakav se često pojavljivao u hrvatskoj komediji dell'arte, Bollé je [...] prisutan kao umjetnik [...] ('Invece che in qualità di creatore affamato dipendente da commissioni arroganti, frequente nella commedia dell'arte croata, Bollé è [...] presente come artista [...]').

È chiara l'allusione alla condizione misera degli artisti che lavorano su commissioni da parte dei «pezzi grossi» e diventano così un personaggio nella commedia dell' arte croata.

5.2. Il secondo italianismo di carattere letterario è una citazione tanto nota che è superfluo qualsiasi commento: arrivata alla metà circa della biografia dell'architetto $\mathrm{D}$. Ibler, Željka Čorak intitola il relativo capitolo Nel mezzo del cammin (UFZ 87).

6. Due italianismi appartengono al livello superiore alla parola: sono un sintagma (4) e una breve frase (5). Entrambi si trovano nel volume OPGM e precisamente nel capitolo Djeca raja ('I bambini del paradiso'). Il titolo allude senz'altro al noto film francese, ma qui è attualizzato in un significato particolarmente amaro: si tratta cioè di una severa critica dei fattori internazionali e della loro ipocrisia in fatto delle 
gravidanze forzate conseguenza degli stupri commessi durante la guerra in Croazia e in Bosnia. Leggiamo:

4) Smrti su postale banalne. Svako toliko netko mora biti raspet, dade mu se humanitarna spužva namočena u ocat, a zatim se ide na ručak. Silovanja su nešto kao začini na medijskom jelovniku, malo žešći, malo ljući, humanitarna problematika a luci rosse, sado-mazo žanr za večernje termine. ('Le morti sono diventate banali. Ognitanto qualcuno deve essere crocifisso, gli si porge la spugna imbevuta d'aceto e poi si va a pranzo. Gli stupri sono qualcosa come condimenti nel menù dei mass media, un po' più forti, un po' più piccanti, problematica umanitaria $a$ luci rosse, genere sado-maso per le ore serali.') (OPGM 35).

Si sa che cinema, locale, sala a luci rosse sono 'quelli riservati esclusivamente alla proiezione di film pornografici' (Zingarelli 1992, ss. vv. luce e rosso); l'allusione è dunque chiara e quanto mai efficace.

5) Svakako, nije riječ ni o čemu novom. Samo o poglavlju u kojemu se nalazimo i u kojemu struktura silovanja jednako pogađa silovanu ženu $i$ silovanu zemlju [...] Već se iz bogatih zapadnih demokracija javljaju usvojitelji djece začete zločinom. Si dà il caso da na tu djecu imaju očinsko pravo. ("Comunque, non si tratta di nulla di nuovo. Soltanto del capitolo in cui ci troviamo e nel quale la struttura dello stupro colpisce ugualmente la donna violentata e il paese violentato. [...] Dalle ricche democrazie occidentali già si fanno vivi degli adottatori dei bambini concepiti nel crimine. Si dà il caso che a questi bambini hanno il patrio diritto.') (OPGM 37).

L'amara ironia risulta dal contrasto, addirittura conflitto, del significato di sporadicità della locuzione si dà il caso (Zingarelli 1992, s. v. caso: 'succede, accade') con la responsabilità implicita dei fattori internazionali.

7. Ed eccoci, infine, arrivati alla componente dialettale del nostro corpus, che è beninteso veneta. $\mathrm{Ci}$ sono cinque venetismi.

7.1. Abbiamo già menzionato la figura con gli angeli. Poiché, dice Željka Čorak, sia la scuola che lo studio universitario le hanno sistematicamente inculcato il disgusto ed il disprezzo (l'autrice usa il verbo zagaditi 'inquinare') per il kitsch, lei ha tenuto la figura a lungo addirittura nella dispensa, e continua:

6) I tako su oni trajali dok mi nije počeo puhati maestral iz te zagađene vale. ('E così essi rimasero là finché cominciò a soffiarmi il maestrale da questo golfo inquinato.') (K 160).

Nel passo, tutto metaforico, troviamo due venetismi diffusi lungo tutta la nostra costa: maestral 'maestrale' (Rosamani 1990: maestral 'maestrale (vento di nord-ovest)'; Doria 1987: maïstral 'maestrale') e vala, ven. vale e val, ital. stand. valle (Rosamani 1990: ss. vv. val e vale, anche 'insenatura del mare'; Doria 1987: s. v. vale 'valle')..$^{5}$ 
7.2. Descrivendo un edificio moderno (UFZ 45) l'autrice oppone le škure ('scuri, scuretti') definite frivole, alle rolete ('persiane a saracinesca') qualificate di puritane. Il sostantivo škure è indubbiamente veneto, ma vi è di genere maschile (Rosamani $1990 \mathrm{e}$ Doria 1987: s. v. scuro, nelle solite accezioni).

7.3. Nello stesso libro (UFZ 191) il tratto di marciapiede sormontato da una sporgenza del piano superiore viene citato come "sottoportego" (sic; virgolette dell'autrice). Anche qui l'origine veneta è sicura (Rosamani 1990: ss. vv. pòrtego e pòrtigo; Doria 1987: s. v. pòrtigo, con la variante pòrtego a Capodistria).

7.4. Appartiene alla componente lessicale veneta anche la seguente voce, con la quale chiudiamo la nostra rassegna. Questa volta abbiamo da fare con la fantasia di Željka Čorak: nel gracidio delle rane, cioè, le pare di scorgere la parola suk (K $82 \mathrm{e}$ 145), ed ecco la spiegazione:

7) Trebalo mi je mnogo godina da shvatim kako je »suk« zapravo »sug«, „šug«, jedna od onih riječi kojima u ovaj kraj [...] dopire more, Hrvatsko Primorje, Italija, Venecija... ('Mi ci vollero molti anni per capire che «suk» in realtà è «sug», «šug», una di quelle voci con le quali fino a queste parti [...] arriva il mare, il Littorale Croato, l'Italia, Venezia...') (K 82).

La componente onomatopeica ci sembra qui alquanto dubbia, ma l'origine della «parola» delle rane è chiara: è il nome sugo, con la variante sug, citato da Rosamani (1990) e da Doria (1987), s. v. sugo (Rosamani: sug in tergestino; Doria: sug senza localizzazione).

8. Con le precedenti pagine abbiamo tentato di illustrare i principali valori degli italianismi nelle opere di Željka Čorak. ${ }^{6}$ Gli elementi italiani appartengono a più settori lessicali: sono termini tecnici, voci quotidiane, elementi affettivi, dialettismi veneti. Essi svolgono varie funzioni affettive, stilistiche, pragmatiche, testuali; ma tutti, in qualsiasi funzione $\mathrm{e}$ in qualunque testo, portano l'inconfondibile stampo della

5 La parola vala è certamente di origine veneta, dunque seriore; se non altro, perché nei romanismi croati antichi la /v/ iniziale è di regola sostituita dalla /b/: VALLU + Bol, VEGIA + (Karlo)bag, VIRGATU + Brgat, probabilmente anche VINCA + bek(v)a 'salice' ecc. Siamo dunque in sostanza d'accordo con il Rocchi (1990, p. 354), eppure le sue parole a proposito dello Skok vanno ridimensionate e corrette. Infatti, dice il Rocchi: «Molto probabilmente non si tratta di un dalmatismo, come vuole lo Skok [...]». Ora, non è che lo Skok vuole vedere in vala un dalmatismo, bensì egli si limita ad accennare alla possibilità dell'origine dalmatoromanza: Može biti dalmato-romanski leksički ostatak ('Può essere un relitto lessicale dalmato-romanzo') (ERHSJ, s. v. vala), il che è tutt'altra cosa.

6 Soltanto dopo la redazione definitiva del presente contributo abbiamo avuto la possibilità di spogliare anche la raccolta di saggi e critiche di Željka Čorak, intitolata Kaleidoskop ('Caleidoscopio') e apparsa nel 1970 a Zagabria. A parte italianismi come «integrati» e "apocalittici» (inseriti come citazioni delle opere di U. Eco; p. 45), vi abbiamo trovato soltanto due italianismi della nostra autrice: i sostantivi loggia, nell'accezione comune (p. 113), e osatura ('ossatura'), nel significato approssimativo di 'struttura portante di un edificio' (pp. 109, 110 e 119). 
personalità dell' autrice. Nel contempo, i suoi italianismi sono altrettanti tasselli del mosaico che attesta la presenza della cultura italiana nelle nostre parti.

\section{Bibliografia}

A. Opere di Željka Čorak (corpus):

U funkciji znaka. Drago Ibler i hrvatska arhitektura između dva rata, SNL i Društvo povjesničara umjetnosti, Zagreb 1981 (UFZ).

Katedrala i XIX. stoljeće, in: Ana Deanovic - Željka Čorak, Zagrebačka katedrala, Globus / Kršćanska sadašnjost, Zagreb 1988, pp. 257-304 (testo) e 367-369 (leggende delle fotografie numm. 305-361) (ZK).

Krhotine. Prilog poznavanju hrvatske provincije u devetnaestom stoljeću, Grafički zavod Hrvatske, Zagreb 1991 (K).

Oproštajno pismo gospodinu Mitterrandu, Durieux, Zagreb 1993 (OPGM).

Zagreb, pisani prostor, Nakladni zavod Matice hrvatske / Mladost, Zagreb 1994 (ZPP).

B. Altri titoli:

Devoto-Oli 1971: G. Devoto - G. C. Oli, Dizionario della lingua italiana, Firenze.

Doria 1987: M. Doria, Grande dizionario del dialetto triestino storico etimologico fraseologico, Trieste.

Rocchi 1990: L. Rocchi, Latinismi e romanismi antichi nelle lingue slave meridionali, Udine.

Rosamani 1990: E. Rosamani, Vocabolario giuliano, Trieste (I edizione 1958, Bologna).

Skok ERHSJ: P. Skok, Etimologijski rječnik hrvatskoga ili srpskoga jezika I-IV, Zagreb 1971-1974.

Zingarelli 1992: Il Nuovo Zingarelli, Vocabolario della lingua italiana, 11. edizione a cura di Miro Dogliotti e Luigi Rosiello, Bologna.

Povzetek

ITALIANIZMI V DELIH ŽELJKE ČORAK

\footnotetext{
Prispevek obravnava italijanske besedne prvine v petih tekstih znane hrvaške umetnostne zgodovinarke, pisateljice in podpredsednice hrvaškega PEN-a. To so: predvsem prelepa knjiga avtoričinih spominov Krhotine, tri knjige arhitektonsko-urbanistične tematike in ena zbirka aktualnih političnih esejev. $\mathrm{V}$ tem korpusu je avtor zasledil kakih dvajset italianizmov (strokovni izrazi, besede splošnega pogovornega jezika, kratke fraze, dialektizmi), v različnih funkcijah (stilistični, pragmatični, tekstualni) in zmeraj močno afektivno obarvanih. Članek ilustrira vsebinske in jezikovno-stilske kvalitete Željke Čorak, obenem je pa tudi delček mozaika italijanske kulture $v$ naših krajih.
} 